\title{
Cotrimoxazol en infecciones óseas: toxicidad e impacto clínico y económico
}

\author{
Alberto Fica, César Lamas, Felipe Olivares, Diego Ramírez, Andrés Soto, \\ Lorena Porte, Stephanie Braun e Ignacia Villablanca
}

\section{Cotrimoxazole in bone-related infections: toxicity and clinical and economic impact}

Background: Cotrimoxazole is a therapeutic option for bone-related infections but is associated to hyperkalemia and renal failure. Tolerance to this drug may reduce length of stay (LOS) and hospital charges. Aims: To evaluate renal, potassium toxicity, clinical outcome, and use of hospital resources in patients treated with cotrimoxazole for bone-related infections. Methods: Retrospective analysis of adult patients with bone-related infections confirmed by culture and treated with this drug. Serum potassium and creatinine levels were analyzed during follow-up and risk factors for hyperkalemia were searched. Length of stay (LOS) and hospital charges were compared. Clinical outcome was evaluated as a secondary endpoint. Results: From 2011 to 2014, 23 patients were identified (mean age 64.7 years). Diabetes mellitus, peripheral vascular disease, and previous amputations prevalence were high $(82.6 \%, 47.8 \%$, and $43.5 \%$, respectively). Median serum potassium concentration increased significantly at first control $(4.35 \mathrm{mEq} / \mathrm{L}$ to $4.9 \mathrm{mEq} / \mathrm{L} ; \mathrm{p}<0.001)$, and also creatinine serum concentration $(0.9$ to $1.1 \mathrm{mg} / \mathrm{dL}$; $\mathrm{p}<0.05)$. Seven patients developed hyperkalemia. Cotrimoxazole was discontinued in 10 patients $(43.5 \%)$, and in 6, discharge was postponed. Drugs active against the renin-angiotensin system (DAARAS) were associated with kyperkalemia (OR $10.8 \mathrm{IC}_{95} 1.37-85 ; \mathrm{p}<0.05$ ). LOS was higher among patients with cotrimoxazole toxicity (median LOS 56 versus 30 days, $\mathrm{p}<0.05$ ). Patients with no cotrimoxazole interruption had less drug-related hospital charges (median values of 563 versus 2820 USD, respectively; $\mathrm{p}<0.01$ ). Conclusions: Cotrimoxazole use must be monitored in order to detect hyperkalemia or renal toxicity and suspend its prescription. Patients that use DAARAS have a higher risk of kyperkalemia. LOS and drug-related hospital charges are reduced when patients can tolerate cotrimoxazole.

Palabras clave: Cotrimoxazol; hyperkalemia, sistema renina-angiotensina, estadía hospitalaria, osteomielitis.

Key words: Cotrimoxazole; hyperkalemia; renin angiotensin system; length of stay, hospital charges, osteomyelitis.

\section{Introducción}

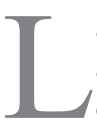
as infecciones óseas en pacientes adultos requieren antibioterapia prolongada para asegurar la erradicación microbiológica y evitar la recurrencia. Es deseable que este tratamiento se aplique por vía oral para facilitar el alta y reducir costos. Estas infecciones son típicas en casos de osteomielitis asociada a pie diabético y en infecciones prolongadas asociadas a prótesis articulares y material de osteosíntesis. Cotrimoxazol es una alternativa terapéutica atractiva en estas condiciones debido a su presentación oral, penetración ósea, cobertura sobre enterobacterias y aislados susceptible o resistentes de Staphylococcus, eficacia razonable y toxicidad conocida ${ }^{1,2}$. Además, su costo es marcadamente inferior a otras alternativas como glicopéptidos o linezolid oral y estas dos, no tienen actividad sobre bacilos gramnegativos. Más aún, no existe cobertura financiera en Chile para ellos y posiblemente también en muchos países del mundo y su adquisición está más allá de la capacidad de compra de los pacientes en Chile, considerado un país de ingresos altos. Esto determina que muchos pacientes deben permanecer hospitalizados para completar un tratamiento prolongado, que habitualmente debe durar 8 a 12 semanas en estos casos ${ }^{3}$. Por otra parte, cotrimoxazol puede provocar falla renal y/o hiperkalemia en adultos mayores, pacientes con diabetes mellitus o falla renal previa y en pacientes que reciben fármacos activos sobre el sistema renina-angiotesina (FASRA), espironolactona o si se usan altas dosis del fármaco ${ }^{4-7}$. Estas condiciones o terapias concomitantes no son infrecuentes en pacientes adultos afectados por infecciones óseas y se desconoce en qué magnitud, esta población puede tolerar este compuesto por los prolongados períodos necesarios en estos casos o en caso contrario, requiere suspenderlo aumentado la estadía hospitalaria y costos asociados. Por lo tanto, una evaluación de la toxicidad de cotrimoxazol en este escenario es relevante, especialmente cuando
Hospital Militar de Santiago Servicio de Infectología (AF, FO, $\mathrm{DR}, \mathrm{AS})$

Laboratorio Microbiología (LP, SB). Equipo de Cirugía Vascular (IV) Hospital DIPRECA, Santiago $(C L)$

Fuente de financiamiento: no hubo Conflictos de interés: ninguno.

Recibido: 4 de mayo de 2015 Aceptado: 6 de octubre de 2015

Correspondencia a: Alberto Fica Cubillos albertofica@gmail.com 
hay bacterias resistentes involucradas. Así, se diseñó un estudio retrospectivo cuyos objetivos fueron evaluar la toxicidad renal y electrolítica de cotrimoxazol, los factores asociados a estas complicaciones, el impacto económico que tiene la suspensión del fármaco por problemas de tolerancia y el desenlace clínico de los pacientes tratados por estas infecciones.

\section{Materiales y Métodos}

\section{Antecedentes}

Desde el año 2011, cotrimoxazol oral ha sido usado progresivamente como una alternativa para el manejo de las infecciones óseas en pacientes adultos en nuestro hospital utilizando un protocolo local. El momento de inicio de cotrimoxazol es decidido por cada equipo tratante y generalmente ocurre después de una intervención quirúrgica, estabilización clínica y disponibilidad de los cultivos tomados. Integrantes del equipo de Infectología asesoran sobre esta posibilidad y la razón de su uso desde el año 2011 obedece a la consolidación de este grupo desde entonces.

\section{Escenario y diseño del estudio}

Estudio retrospectivo desarrollado en el Hospital Militar, un centro de referencia de 300 camas localizado en Santiago, Chile. Los objetivos primarios fueron evaluar el desarrollo de toxicidad renal e hiperkalemia en pacientes tratados con cotrimoxazol para infecciones ósea, los factores asociados a este desenlace, y la estadía y gastos hospitalarios en pacientes que presentan o no presentan toxicidad. Como objetivo secundario se evaluó la eficacia clínica.

\section{Selección de casos}

Se incluyeron como casos pacientes con osteomielitis, asociada o no asociada a pie diabético e infecciones asociadas a prótesis articular o material de osteosíntesis. Todos los casos, excepto uno en el que se tomó una muestra de cultivo desde una fístula crónica, tuvieron muestras microbiológicas directas desde hueso o articulación (en caso de prótesis articular) obtenidas por técnica estéril durante un procedimiento quirúrgico. No se utilizó un criterio histopatológico.

Esta serie incluye pacientes con al menos un aislado bacteriano susceptible a cotrimoxazol, tratados entre los años 2011 y 2014. La dosis de cotrimoxazol fue definida por el equipo tratante. Se incluyeron sólo casos con registros suficientes sobre las razones para el inicio del compuesto y su suspensión si fuera el caso, y con al menos una determinación de kalemia o creatininemia de seguimiento, además del valor basal.

\section{Recolección de datos}

Las fichas médicas fueron revisadas para obtener información demográfica, co-morbilidades incluyendo diabetes mellitus tipo 2 , retinopatía diabética, cardiopatía, tabaquismo actual o previo, enfermedad vascular periférica o coronaria, revascularización coronaria o periférica, amputación previa de extremidades, enfermedad renal crónica, tipo de infección ósea y procedimientos quirúrgicos o vasculares efectuados durante la hospitalización actual. También se rescató la información microbiológica y susceptibilidad antimicrobiana.

\section{Evaluación de la toxicidad renal e hiperkalemia}

En la versión actual del protocolo, se obtienen valores basales de nitrógeno ureico en sangre (NU0), kalemia (K0) y creatininemia $(\mathrm{C} 0)$ con un seguimiento cada 3 a 5 días después de iniciar cotrimoxazol. Cuando al menos dos valores de seguimiento consecutivo (NU1-NU2; K1$\mathrm{K} 2$; C1-C2) no demuestran un aumento sobre el rango $\operatorname{normal}(\mathrm{NU}>23 \mathrm{mg} / \mathrm{dL} ; \mathrm{C}>1,1 \mathrm{mg} / \mathrm{dL}$ y K $>5,5 \mathrm{mEq} / \mathrm{L}$ ), se autoriza el alta del paciente y su uso prolongado. La interrupción de este compuesto es sugerida cuando se observa un aumento más allá de los valores normales, aún en controles tardíos, pero los médicos a cargo pueden continuar la terapia con nuevos seguimientos de laboratorio si los aumentos son discretos en relación al valor máximo normal. Para evaluar toxicidad, los valores absolutos de kalemia, creatinina plasmática y NU fueron analizados desde el momento basal al último control disponible y se calcularon los porcentajes de interrupción de terapia por valores anormales. Además, se exploraron potenciales factores asociados a un aumento de estos parámetros (ver sección análisis estadístico). La presencia de injuria renal aguda (IRA) fue reconocida según lo descrito previamente. Brevemente, por incrementos paralelos de creatininemia y NU y usando diferentes criterios dependiendo de si el paciente tiene falla renal crónica o $\mathrm{no}^{4}$.

\section{Análisis de la estadía y gastos hospitalarios entre pacientes con y sin interrupción de cotrimoxazol}

Esta comparación se realizó en aquellos pacientes que iniciaron cotrimoxazol durante la hospitalización, midiendo específicamente su estadía hospitalaria y los gastos hospitalarios totales y por fármacos, información gentilmente provista por la Unidad del Bioestadística del hospital. Los gastos fueron actualizados a diciembre de 2014 usando la herramienta respectiva disponible en la página web del Instituto Nacional de Estadística de Chile (disponible en http://encina.ine.cl/calculadora) y transformados a USD según la paridad cambiara de ese mes.

\section{Eficacia clínica comparativa}

La eficacia clínica entre pacientes que continuaron con cotrimoxazol u otros compuestos, fue evaluada por 
la tasa de re-intervenciones quirúrgicas y recurrencia de infección al último punto de seguimiento.

\section{Aprobación por Comité de Ética y análisis estadístico}

Debido al pequeño tamaño de la serie, sólo se aplicaron métodos no paramétricos. Las variables categóricas fueron comparadas por la prueba bilateral de Fisher y las variables continuas por la prueba de Mann Whitney en el caso de grupos independientes y por la prueba de Wilcoxon para muestras pareadas en caso de valores secuenciales. La búsqueda de factores de riesgo para hiperkalemia fue desarrollada en tablas de contingencia con prueba de Fisher y cálculo de odds ratio (OR). Se estableció un valor $\mathrm{p}<0,05$ como estadísticamente significativo. Esta investigación fue aprobada por el Comité de Ética institucional.

\section{Resultados}

\section{Características de la población tratada con cotrimoxazol}

Durante los cuatro años de este estudio, se identificó a 23 pacientes tratados con cotrimoxazol con información suficiente para análisis: 16 casos con osteomielitis asociada a pie diabético $(69,6 \%)$, cuatro por infección asociada a prótesis articular o material de osteosíntesis $(17,4 \%)$ y tres por osteomielitis de otras causas. La mayor parte de los pacientes era de sexo masculino (n: $18 ; 78,3 \%$ ) y la edad promedio fue de 64,7 años (rango 32-93). La prevalencia de diabetes mellitus tipo $2(82,6 \%)$, enfermedad vascular periférica $(47,8 \%)$, enfermedad renal crónica $(30,4 \%)$ y tabaquismo fue alta $(30,4 \%)$, al igual que de cardiopatía $(21,7 \%)$. Además, $43,5 \%$ tenía amputación previa de causa vascular (Tabla 1). La antigüedad promedio del diagnóstico de diabetes mellitus fue de 15,4 años y 55\% tenía retinopatía diabética.

\section{Datos microbiológicos}

Los cultivos fueron monomicrobianos en 17 casos $(73,9 \%)$ y polimicrobianos en el resto $(26,1 \%)$ totalizando 29 aislados (Tabla 2). Incluyendo dos aislados de Enterococcus faecalis que tienen resistencia intrínseca a cotrimoxazol, la mayor parte de los aislados fueron microorganismos grampositivos (26 de 29;89,7\%), y dentro de este grupo, Staphylococcus aureus fue predominante (n: 10; 38,5\%). El grupo Staphylococcus coagulasa negativa representó la mayor parte de los grampositivos (n: $13 ; 50 \%$ ) y se identificó un aislado de Kocuria spp. $(3,8 \%)$. En total, 16 de 26 aislados grampositivos fueron resistentes a meticilina (66,7\%). Además, se identificaron tres aislados de enterobacterias, todos ellos susceptible a cotrimoxazol (Tabla 2).
Tabla 1. Características basales de 23 pacientes tratados con cotrimoxazol para infecciones óseas, Hospital Militar de Santiago, 2011-2014

\begin{tabular}{|lcc|}
\hline Parámetro & n/N & Resultado \\
Edad promedio (rango) en años & & $64,7(32-93)$ \\
Sexo masculino & $18 / 23$ & $78,3 \%$ \\
\hline Hipertensión arterial & $13 / 22$ & $59,1 \%$ \\
\hline Diabetes mellitus tipo 2 & $19 / 23$ & $82,6 \%$ \\
\hline Antigüedad del diagnóstico en años (rango) & $17 / 23$ & $15,4(5-36)$ \\
\hline Retinopatía diabética & $11 / 20$ & $55,0 \%$ \\
\hline Enfermedad arterial oclusiva periférica & $11 / 23$ & $47,8 \%$ \\
\hline Amputación secundaria a causa vascular & $10 / 23$ & $43,5 \%$ \\
\hline EPOC u otra condición pulmonar crónica & $1 / 23$ & $4,3 \%$ \\
\hline Revascularización coronaria & $2 / 23$ & $8,7 \%$ \\
\hline Intervención vascular periférica & $5 / 23$ & $21,7 \%$ \\
\hline Cardiopatía & $5 / 23$ & $21,7 \%$ \\
\hline Insuficiencia cardíaca & $2 / 23$ & $8,7 \%$ \\
\hline Enfermedad renal crónica & $7 / 23$ & $30,4 \%$ \\
\hline Tabaquismo previo & $11 / 23$ & $47,8 \%$ \\
\hline Tabaquismo actual & $7 / 23$ & $30,4 \%$ \\
\hline EPOC: enfermedad pulmonar obstructiva crónica. & & \\
\hline
\end{tabular}

Tabla 2. Distribución de microorganismos obtenidos de muestras tisulares en 23 pacientes con infecciones óseas. Hospital Militar de Santiago, 2011-2014

\section{Microorganismo}

n

Grampositivos

Staphylococcus aureus: susceptible a meticilina (SASM)

Staphylococcus aureus: resistente a meticilina (SARM)

Staphylococcus epidermidis: resistente a meticilina

Staphylococcus simulans: susceptible a meticilina

Staphylococcus capitis: resistente a meticilina

Staphylococcus caprae: resistente a meticilina

Staphylococcus haemolyticus: resistente a meticilina

Staphylococcus coagulasa negativa: resistente a meticilina

Kocuria spp. (sin estudio de susceptibilidad)

Enterococcus faecalis (no susceptible a cotrimoxazol)

Total de grampositivos resistentes a meticilina

Total de grampositivos (incluyendo Enterococcus faecalis)

\section{Gramnegativos}

Citrobacter freundii

Klebsiella pneumoniae

Proteus mirabilis

Total
$\%$

8,7

66,7

89,7

\section{Terapia con cotrimoxazol}

Fue indicado para el manejo de infecciones por grampositivos en 21 pacientes $(91,7 \%)$ y por gramnegativos en dos casos $(8,7 \%)$. En la mayoría de nuestros pacientes, el tratamiento fue iniciado durante la hospitalización (n: 21; $91,3 \%$ ) y la prescripción antimicrobiana fue indicada por 
una duración precisa en 19 casos $(82,6 \%)$ y como terapia de supresión por persistencia de prótesis o material de osteosíntesis en los cuatro casos restantes $(17,4 \%)$. En sólo cuatro casos $(17,4 \%)$, se usó terapia combinada ya fuese

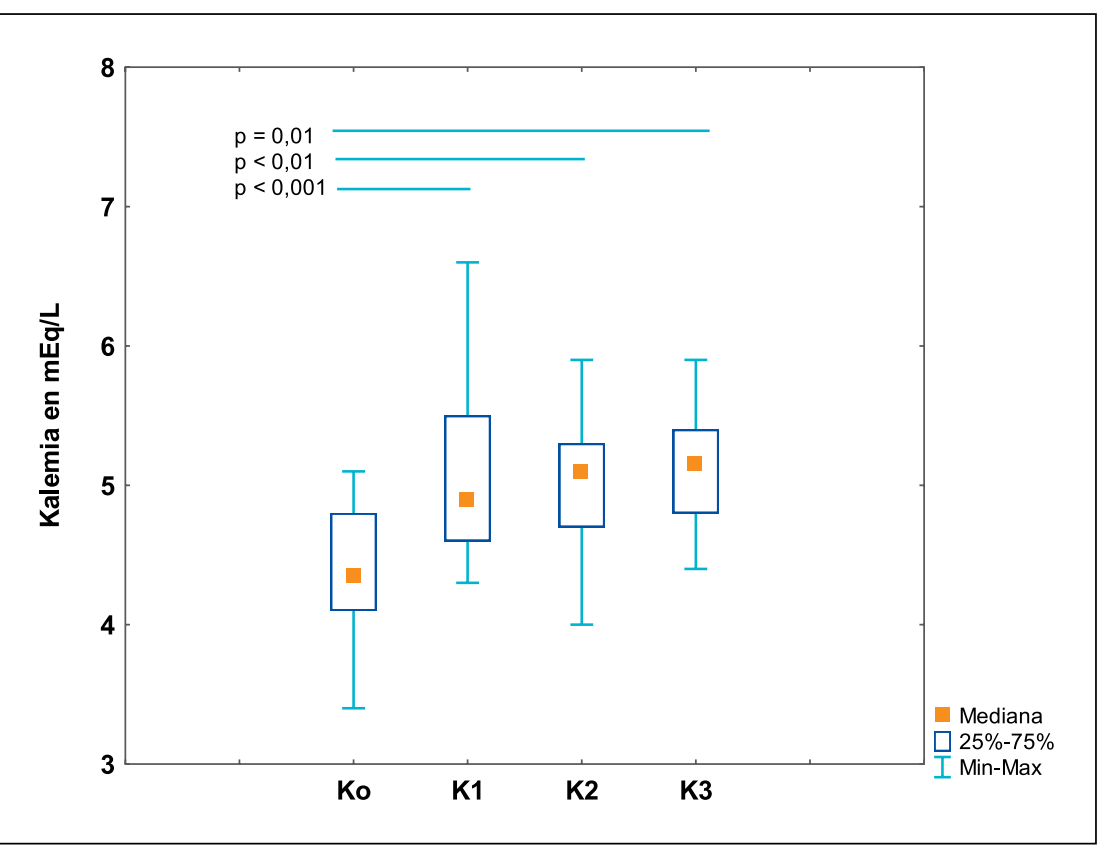

Figura 1. Mediana, valor mínimo y máximo y rango interquartil (25-75\%) de la kalemia basal (K0) y a diferentes momentos de seguimiento (K1 a K3) en pacientes tratados con cotrimoxazol.

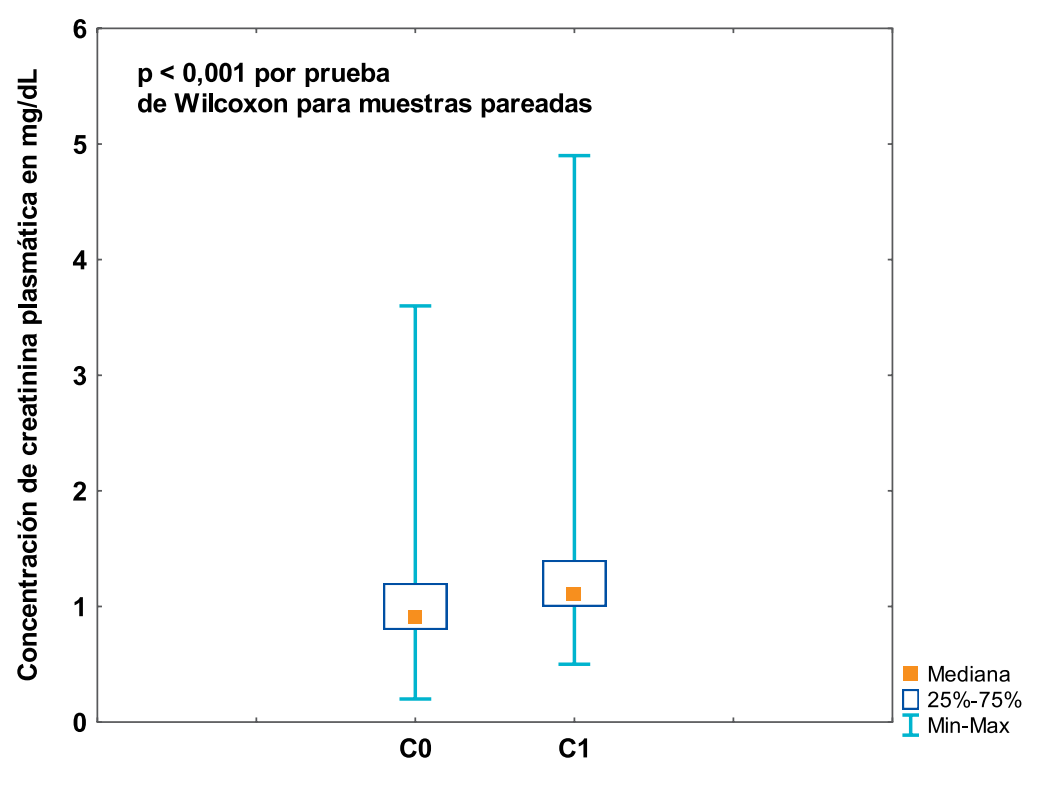

Figura 2. Mediana, valor mínimo y máximo y rango interquartil (25-75\%) de la creatininemia basa (C0) y al primer control (C1) en pacientes tratados con cotrimoxazol. con rifampicina (n: 2) o con amoxicilina, en este último caso para cubrir E. faecalis (n: 2). La mayor parte de los casos tuvo un tratamiento quirúrgico asociado, ya fuese como amputación o aseo (n: 22; 95,7\%) y en dos casos como procedimiento vascular intervencional $(8,7 \%)$. La dosis promedio de cotrimoxazol fue 2,4 tabletas forte por día (rango 1-4).

Nota del Editor: Cotrimoxazol forte $=$ sulfametoxazol $800 \mathrm{mg}+$ trimetoprim $160 \mathrm{mg}$.

\section{Cambios en concentración de potasio, creatininemia y NU en plasma o suero durante la terapia}

Excluyendo a un caso aislado en el que se tomó el primer control de kalemia a los 48 días de iniciar la terapia, K1 fue evaluado entre 2 y 7 días (promedio 3,6 días) después del inicio de cotrimoxazol. La mediana de $\mathrm{K} 0$ fue $4,35 \mathrm{mEq} / \mathrm{L}$, cifra que aumentó significativamente a 4,9 mEq/L en K1 ( $p<0,001$ por test de Wilcoxon para 20 pares de observaciones (Figura 1). El cambio promedio entre $\mathrm{K} 0$ y K1 fue $0,8 \mathrm{mEq} / \mathrm{L}$ (rango $-0,2 \mathrm{a}+2,5 \mathrm{mEq} / \mathrm{L}$ ). La kalemia también aumentó significativamente durante K2 (tomado con una mediana de 4 días después de K1) respecto a los valores basales ( $\mathrm{p}<0,01$ para 13 pares de comparaciones) y en K3 (p: 0,01 para 9 pares de observaciones, tomado con una mediana de 16 días post $\mathrm{K} 2$ ). En contraste, las cifras en K2 no fueron significativamente diferentes de $\mathrm{K} 1$ (mediana $5,1 \mathrm{mEq} / 1$ versus 4,9 $\mathrm{mEq} / \mathrm{L}$, respectivamente) ni en $\mathrm{K} 3$ respecto a $\mathrm{K} 2$ (Figura 1), demostrando estabilidad después del ascenso inicial. En esta serie, siete pacientes desarrollaron hiperkalemia $(>5,5 \mathrm{mEq} / \mathrm{L})$ durante el tratamiento $(30,4 \%)$.

Las concentraciones de creatininemia también aumentaron significativamente en $\mathrm{C} 1$, aunque este aumento fue discreto. Una mediana en $\mathrm{C} 0$ de $0,9 \mathrm{mg} / \mathrm{dL}$ aumentó a 1,1 $\mathrm{mg} / \mathrm{dL}$ en $\mathrm{C} 1(\mathrm{p}<0,05)$ (Figura 2$)$. El aumento promedio entre $\mathrm{C} 0$ y $\mathrm{C} 1$ fue de $0,17 \mathrm{mg} / \mathrm{dL}$ (rango $-1,4 \mathrm{a}+1,31$ ). Los valores de NU no aumentaron significativamente durante el seguimiento (datos no mostrados). Dos de 22 pacientes $(9,1 \%)$ con información disponible evolucionaron con IRA aguda, según criterios predefinidos.

Trece pacientes $(56,5 \%)$ recibieron fármacos concomitantes que pudieron haber influenciado la kalemia o creatininemia: ocho $(34,8 \%)$ recibieron FASRA; cinco $(21,7 \%)$ anti-inflamatorios no esteroidales; tres (13\%) carvedilol; dos $(8,7 \%)$ diuréticos; dos $(8,7 \%)$ espironolactona; uno (4,3\%) antimicrobianos nefrotóxicos y otros $(4,3 \%)$ medio de contraste.

\section{Discontinuación de cotrimoxazol y suspensión del alta hospitalaria}

En 10 pacientes $(43,5 \%)$ la terapia con cotrimoxazol fue discontinuada debido a un aumento más allá de los valores normales de las concentraciones de potasio o 
creatininemia: en cinco casos $(21,7 \%)$ por hiperkalemia, en tres por incrementos de creatininemia $(13 \%)$ y en otros dos por ambos parámetros $(8,7 \%)$. Esta interrupción ocurrió al primer control en cinco casos $(50 \%)$, después del segundo control en dos $(20 \%)$, después del tercero en dos $(20 \%)$ y después del cuarto en el caso restante (10\%). En seis de estas interrupciones (26,1\%), se postergó el alta hospitalaria, los pacientes fueron traspasados a terapia con linezolid oral y se iniciaron esfuerzos administrativos para conseguir cobertura financiera para terapia con linezolid luego del alta. La interrupción de la terapia en el resto determinó un cambio a fluoroquinolonas (dos casos, uno post alta) o linezolid en un caso post alta. En un paciente no se prescribió más terapia antimicrobiana.

\section{Factores asociados a hiperkalemia e interrupción de terapia}

El uso concomitante de FASRA fue el único factor de los explorados que se asoció significativamente al desarrollo de hiperkalemia en cualquier control. Cinco de ocho pacientes que usaron estos fármacos desarrollaron hiperkalemia en comparación con dos de 15 que no las usaron (OR 10,8 $\mathrm{IC}_{95} 1,37-85 ; \mathrm{p}<0,05$ por prueba bilateral de Fisher). Por ejemplo, el valor promedio en K1 de ocho pacientes que recibieron estos compuestos fue de $5,5 \mathrm{mEq} / \mathrm{L}$ versus $4,9 \mathrm{mEq} / \mathrm{L}$ en $\mathrm{K} 1$ para aquellos que no recibieron este tipo de terapia $(\mathrm{p}<0,01$ por prueba de Mann Whitney) (Figura 3). Otros factores como edad, diabetes mellitus, hipertensión arterial, enfermedad vascular periférica, cardiopatía, revascularización coronaria o periférica, tabaquismo, enfermedad renal crónica u otros fármacos concomitantes no se asociaron a hiperkalemia. Además, la duración de la diabetes mellitus, los niveles de hemoglobina A glicosilada al ingreso o la dosis diaria de cotrimoxazol, no se asociaron a este resultado. Tampoco pudimos identificar algún factor asociado a un aumento en las concentraciones plasmáticas de creatinina.

\section{Estadía y gastos hospitalarios}

Como se mencionó, en seis de 21 casos que iniciaron terapia durante la hospitalización, el alta fue postpuesta hasta asegurar cobertura financiera para una terapia con linezolid oral. La comparación de este grupo contra los 15 casos restantes que no sufrieron una suspensión de la terapia con cotrimoxazol, demostró que la estadía hospitalaria fue significativamente más prolongada (mediana de 56 versus 30 días, $\mathrm{p}<0,05$ por prueba de Mann-Whitney) (Tabla 3). Por otra parte, aunque los gastos hospitalarios no difirieron entre ambos grupos (medianas de 12.521 vs 9.914 USD, respectivamente), los pacientes sin interrupción de la terapia con cotrimoxazol tuvieron menos gastos por fármacos (mediana de 563 versus 2.820 USD, respectivamente; $\mathrm{p}<0,01)$.

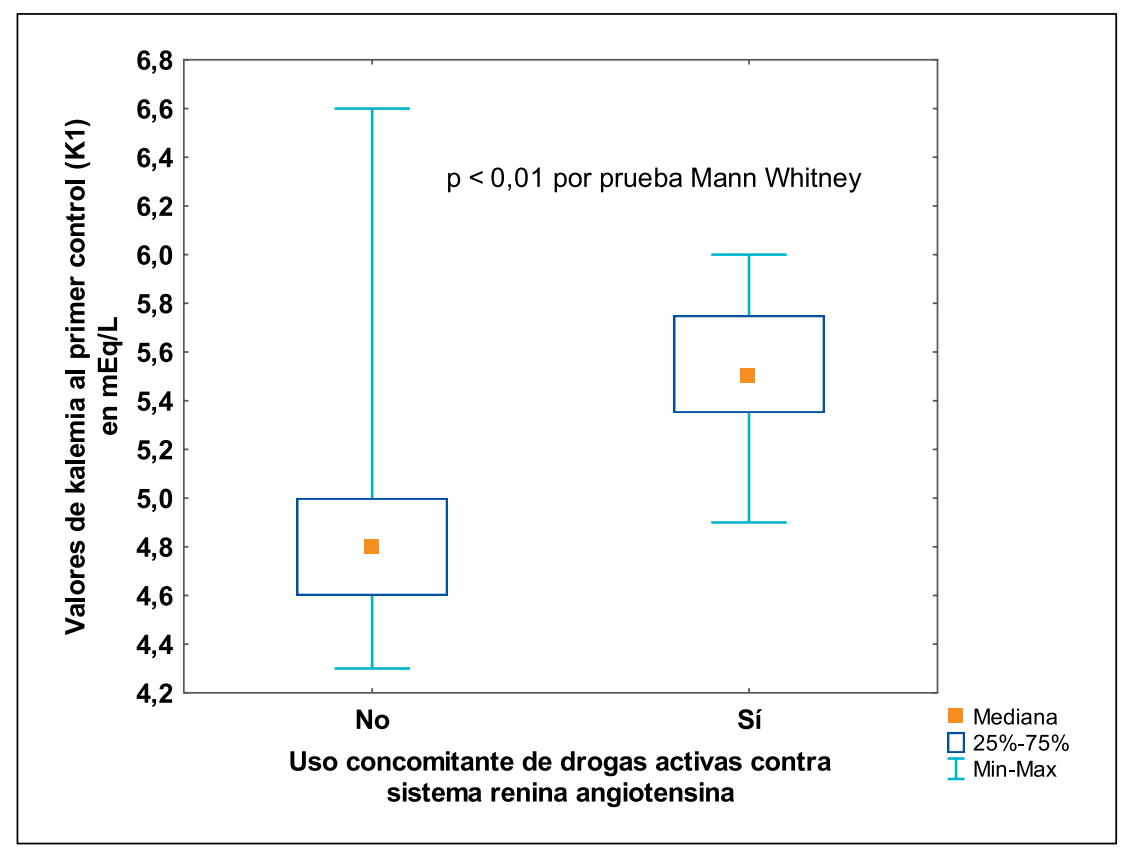

Figura 3. Distribución de la mediana, valor mínimo y máximo y rango interquartil (25-75\%) de los valores de potasio plasmático entre pacientes que recibieron o no recibieron FASRA.

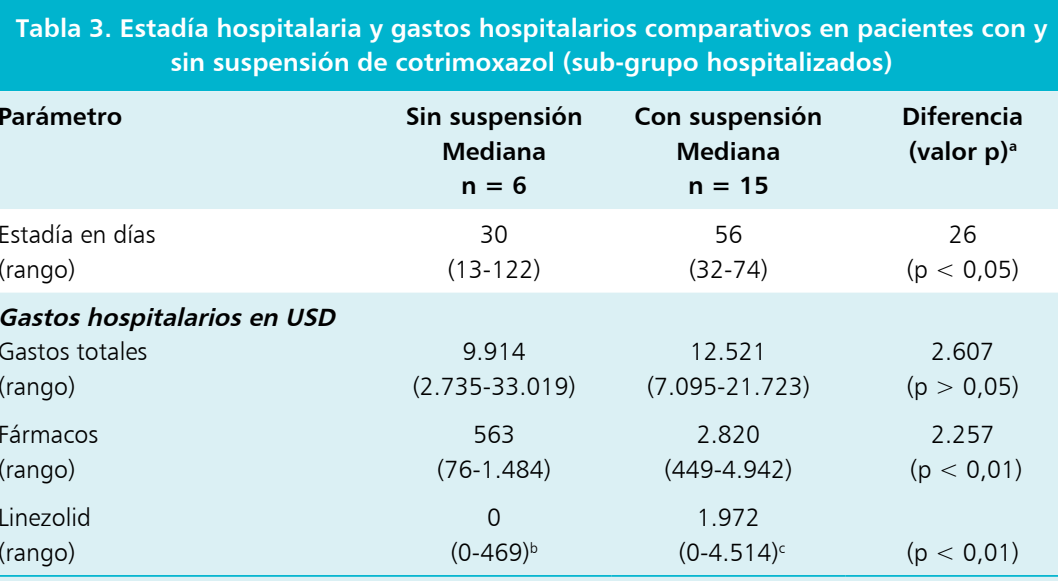

aPrueba de Mann-Whitney. ${ }^{\text {bUn }}$ caso con uso transitorio de linezolid. 'Datos incluyen cuatro casos con linezolid antes del alta.

\section{Evolución y eficacia clínica}

Nueve pacientes $(39,1 \%)$ requirieron nuevas intervenciones quirúrgicas, ya fuese por amputación o aseo. La tasa de intervenciones quirúrgicas fue similar entre los pacientes que interrumpieron o no interrumpieron la terapia con cotrimoxazol (4 de 10 versus 5 de 13). La mediana de seguimiento post alta fue de 160 días (rango 0-906; sólo un paciente perdido). Dos de los cuatro pacientes en terapia de supresión siguen con cotrimoxazol, uno debió 
suspenderlo y pasar a fluoroquinolonas con la que se mantiene y el último, la finalizó al ser cambiada la prótesis articular. Dos recurrencias $(15,4 \%)$ fueron observadas en el grupo que continuó con cotrimoxazol (ambas a los tres meses del alta) y se observó una recurrencia en el grupo que continuó con otro antimicrobiano por intolerancia al cotrimoxazol (10\%, recaída a los cuatro meses del alta), sin ser estas diferencias significativas.

\section{Discusión}

Las infecciones óseas tales como osteomielitis y aquellas asociadas a infecciones crónicas de prótesis o material de osteosíntesis, requieren el control adecuado del foco infeccioso y una antibioterapia prolongada para asegurar la erradicación microbiológica y evitar la recurrencia. Esta terapia debe considerar varios factores que pueden afectar los resultados clínicos incluyendo la penetración tisular, cobertura antimicrobiana, interacciones con otros fármacos, aspectos farmacocinéticos-farmacodinámicos, presencia de material ortopédico, biopelículas, función de órganos, efectos adversos, costo y adherencia al tratamiento.

Cotrimoxazol es una de las alternativas para tratar estas infecciones. La literatura científica disponible originada principalmente de estudios observacionales como éste o estudios comparativos no randomizados, indica que la eficacia clínica oscila entre 45 y $100 \%{ }^{3,8,9}$. La dosis y duración de la terapia, aseos quirúrgicos o remoción de material ortopédico, uso de terapia antimicrobiana combinada con rifampicina o quinolonas, y la duración del seguimiento, explican parte de estos resultados variables $^{3,8,9}$.

Cotrimoxazol tiene efectos adversos conocidos tales como reacciones alérgicas, molestias digestivas, anemia megaloblástica y anemia hemolítica asociada a deficiencia de glucosa-6-fosfato deshidrogenasa. Además, tiene el potencial de aumentar los efectos tóxicos de otros fármacos concomitantes debido a la competencia por los sitios de unión en la albúmina, tales como warfarina, sulfonilureas, diuréticos tiazídicos, fenitoina y metotrexato, medicamentos que no son infrecuentes en adultos mayores o con enfermedades crónicas. Cotrimoxazol también se asocia a nefritis intersticial, leucopenia, neutropenia o trombocitopenia. A pesar que las reacciones alérgicas y las molestias digestivas son reportadas hasta en $20 \%$ de los pacientes adultos que reciben este compuesto por períodos prolongados $^{8,9}$, el desarrollo de hiperkalemia o aumento en la creatininemia ha recibido menos atención ${ }^{4,5,10,11}$. Contribuye a una falsa percepción de seguridad, el uso infrecuente que tiene esta molécula actualmente, la que además es aplicada en bajas dosis y en forma intermitente en casos de profilaxis (Pneumocystis jiroveci, por ejemplo) o por períodos cortos.
La hiperkalemia es explicada por un efecto inhibitorio de trimetoprim sobre los canales de sodio ubicados en la membrana apical en las células principales del epitelio del túbulo colector cortical. Mediante esta inhibición, el influjo de sodio es restringido provocando natriuresis lo que a su vez disminuye la fuerza neta que permite la secreción de $\mathrm{K}+\mathrm{y} \mathrm{H}+$ hacia el lumen. Además, trimetoprim inhibe la bomba $\mathrm{Na}+\mathrm{K}+\mathrm{ATPasa}$ localizada en la membrana basolateral del túbulo colector medular o cortical, previniendo la transfererencia de $\mathrm{K}+$ desde el intersticio hacia el interior de la célula ${ }^{12}$. El impacto de trimetoprim en la homeostasis del potasio es más frecuente que otros efectos secundarios asociados a la natriuresis como hiponatremia, hipovolemia y falla renal o secreción de $\mathrm{H}+(\text { acidosis metabólica })^{12-14}$.

Los factores de riesgo para hiperkalemia incluyen edad mayor, altas dosis de cotrimoxazol, diabetes mellitus, falla renal previa, SIDA, inhibidores de la enzima convertidora de angiotensina y bloqueadores del receptor de angiotensina (FASRA en ambos casos) o espironolactona ${ }^{4,6,7,15}$. Se ha estimado que el uso concomitante de cotrimoxazol con FASRA aumenta seis veces el riesgo de hiperkalemia cuando se compara con amoxicilina, por ejemplo y que esta asociación genera reingresos ${ }^{6}$. Por otra parte, el uso paralelo de espironolactona amplifica el riesgo de hospitalizaciones por hiperkalemia 12 veces $^{16}$. Más grave aún, el uso conjunto de cotrimoxazol con espironolactona o FASRA aumenta el riesgo de muerte súbita en estudios poblacionales, subrayando la relevancia de estas asociaciones ${ }^{17,18}$. Incluso en la población con infección por VIH, un grupo que generalmente no utiliza estos fármacos y no es de edad mayor, las altas dosis de cotrimoxazol usadas para tratar neumonía por $P$. jiroveci provocan hiperkalemia en cerca de $20 \%$ de los pacientes ${ }^{10}$.

La injuria renal aguda (aumento conjunto del NU y creatininemia) apareció en una pequeña fracción de nuestros casos y el porcentaje observado fue similar a lo reportado en un gran estudio retrospectivo con más de 500 pacientes $(11,2 \text { y } 9,1 \% \text {, respectivamente })^{4}$. La IRA parece ser explicada por daño renal intrínseco o nefritis intersticial $^{4}$. En algunos casos, los incrementos aislados de creatininemia son provocados por interferencia del cotrimoxazol en su secreción tubular ${ }^{4}$.

Intentamos caracterizar los problemas del potasio y creatinina en una población extrema al recibir cotrimoxazol para el manejo de infecciones óseas y encontramos varios aspectos que merecen mayor análisis. La hiperkalemia apareció en $30 \%$ de nuestros casos, posiblemente un valor más elevado que lo registrado en población portadora de VIH o adulta mixta donde $20 \%$ presenta esta complicación ${ }^{5,10}$. Creemos que esta cifra mayor es en parte explicada por el perfil de nuestros pacientes, caracterizada por la alta prevalencia de diabetes mellitus, enfermedad 
renal crónica, cardiopatía y uso de fármacos que afectan la homeostasis renal y del potasio. Esta cifra es importante porque debe alertar al médico acerca de la importancia de evaluar factores del hospedero y terapias concomitantes antes de prescribir cotrimoxazol en la población adulta. Además y como ha sido reportado por otros, pudimos relacionar el aumento del potasio al uso paralelo de FASRA $^{6,7,16}$. Aproximadamente un tercio de nuestros pacientes estaban usando estos compuestos, indicando que las co-morbilidades y necesidades del paciente limitan las posibilidades de discontinuarlas, limitando la tolerancia al cotrimoxazol. No obstante, y a pesar de nuestra dañada población, cotrimoxazol fue tolerado por cerca de $60 \%$, facilitando el alta hospitalaria y la reducción de costos. Finalmente, pudimos demostrar que la estadía y los gastos por fármacos fueron significativamente inferiores en el grupo que toleró cotrimoxazol.

El hecho de que sólo $60 \%$ pudo completar la terapia con cotrimoxazol, puede parecer frustrante. Sin embargo, este número es relevante para un país en desarrollo donde el costo en una variable limitante, especialmente en el caso de infecciones por bacterias grampositivas resistentes (cerca de 70\% de nuestros casos). En Chile, la terapia con cotrimoxazol cuesta $<1$ USD al día y el de linezolid alcanza $>80 \mathrm{USD} /$ día. Debido que la tolerancia a cotrimoxazol permite la liberación de camas, la selección de este antimicrobiano también contribuye al ingreso de nuevos pacientes con otras condiciones en un ambiente con listas de espera para ocupar una cama hospitalaria. En este trabajo, la mitad de los pacientes estuvo hospitalizado $\leq 26$ días cuando pudieron tolerar el cotrimoxazol. Así, la prescripción de este compuesto clásico no sólo es más barato sino que también contribuye a la equidad social.

Linezolid tiene interacciones citocromales mínimas o ninguna y alcanza concentraciones óseas adecuadas ${ }^{19}$. En series clínicas o estudios comparativos no randomizados que involucran pacientes con osteomielitis, linezolid ha demostrado tasas de curación entre 55 y $100 \% 0^{20-23}$, valores similares a los obtenidos con cotrimoxazol ${ }^{3,8,9}$. Sin embargo, linezolid es un compuesto caro y se asocia a un riesgo de anemia, trombocitopenia, polineuropatía, neuritis óptica o acidosis láctica cuando se usa por períodos prolongados ${ }^{21,22,24-26}$. Además, puede inducir toxicidad serotoninérgica cuando se aplica con inhibidores de la recaptación de serotonina ${ }^{25}$. Considerando su eficacia comparativa, en un estudio controlado randomizado, cotrimoxazol-rifampicina demostró ser equivalente a linezolid-rifampicina en pacientes con infecciones óseas o articulares, aunque el número de casos con osteomielitis fue pequeño ${ }^{8}$.

Cotrimoxazol no sólo fue usado para el manejo de bacterias grampositivas resistentes sino que también fue considerado en algunos casos con enterobacterias y en forma preferente sobre quinolonas. Estos compuestos, especialmente levofloxacina, y en menor grado moxifloxacina, logran buenas concentraciones tisulares y han sido validados en varias experiencias ${ }^{4}$. Una ventaja de levofloxacina es la posibilidad de usar altas dosis lo que optimiza su efecto farmacodinámico. No obstante, es más caro que cotrimoxazol, tiene interacciones con cationes bivalentes en el lumen intestinal, con otros fármacos a nivel microsomal y el riesgo de prolongar el intervalo QTc con algunos medicamentos. De esta manera, los pacientes con polifarmacia están de alguna manera limitados para recibir en primera instancia terapias con fluoroquinolonas.

En nuestro trabajo no usamos $\beta$-lactámicos para tratar aislados susceptibles. La penetración ósea de cloxacilina oral es muy limitada debido a su baja concentración plasmática $(10 \mu \mathrm{g} / \mathrm{mL})$, alta unión a proteínas $(>90 \%)$ y la naturaleza hidrofílica del compuesto que explica una relación hueso/plasma de sólo $10 \%{ }^{27,28}$. Flucloxacilina tienen mayor biodisponibilidad oral pero no alcanza concentraciones óseas útiles ${ }^{28}$. De esta manera, el tratamiento exitoso de osteomielitis por $S$. aureus parece ser posible sólo con altas dosis de cloxacilina parenteral y por varias semanas ${ }^{29}$.

Este trabajo tiene varias limitaciones. No pudimos reclutar más casos a pesar de los cuatro años de inclusión y de una política establecida para adoptar cotrimoxazol como alternativa, lo que limitó el tamaño de la población y una mejor caracterización estadística. Así, la tendencia observada hacia un menor gasto hospitalario global cuando hay tolerancia al compuesto (medianas de 9.000 vs 12.000 USD aproximadamente), podría haber alcanzado valor estadístico y quizás otros factores, además del uso de FASRA, podrían haber sido identificados como se ha reportado en otras series. Aún así, nuestros resultados están en línea con otras publicaciones y según nuestro conocimiento, son los primeros en reportar la importancia que tiene la prescripción de cotrimoxazol en los gastos hospitalarios en pacientes adultos con infecciones óseas. Se debe enfatizar que la demora administrativa para conseguir terapia antimicrobiana alternativa participó en los mayores gastos en el grupo que no toleró el cotrimoxazol, un hecho que no necesariamente sucede en otros centros de atención. Tampoco incluimos una evaluación económica post egreso pero parece poco probable que los resultados puedan cambiar sustantivamente luego del alta. Asimismo, la evaluación del impacto clínico que se hizo fue modesta debido a que la muestra fue pequeña y porque hay muchos factores que participan en el desenlace final. Sin embargo, no logramos observar que el grupo con cotrimoxazol tuviera un peor resultado que el grupo que debió cambiar terapia. Se debe enfatizar que los resultados clínicos obtenidos están favorecidos por la alta tasa de desfocación efectuada en nuestros pacientes. 
En conclusión, cotrimoxazol es una opción terapéutica para el tratamiento de infecciones óseas en pacientes adultos, incluyendo aquellos con bacterias resistentes. $\mathrm{Su}$ uso debe ser monitorizado para detectar aumentos plasmáticos peligrosos de potasio o creatinina. Esta política es especialmente importante en pacientes mayores o aquellos con co-morbilidades o fármacos que afectan la homeostasis renal y del potasio. Aunque no todos los pacientes finalmente tolerarán este compuesto clásico, es posible anticipar quiénes tendrán estas complicaciones. Al final, una fracción relevante se beneficiará de esta opción, será posible el alta y una reducción de costos.

Agradecimientos: A la Unidad de Bioestadística del Hospital Militar.

\section{Resumen}

Antecedentes: Cotrimoxazol es una alternativa en infecciones óseas pero se ha asociado al desarrollo de falla renal e hiperkalemia. Objetivo: Evaluar toxicidad renal, hiperkalemia, estadía y gastos hospitalarios y evolución clínica en un grupo de pacientes con infecciones óseas tratados con este compuesto. Pacientes y Métodos: Estudio retrospectivo-descriptivo de pacientes adultos con infecciones óseas confirmadas con cultivos y tratados con este compuesto. Seguimiento de creatinina y kalemia y búsqueda de factores de riesgo para hiperkalemia, comparación de gastos y estadía hospitalaria y análisis de eficacia clínica. Resultados: Desde el año 2011 al 2014 se identificaron 23 pacientes (promedio de edad 64,7 años). La prevalencia de diabetes mellitus tipo 2 (82,6\%), enfermedad vascular periférica $(47,8 \%)$ y amputaciones previas $(43,5 \%)$ fue elevada. La mediana de la kalemia basal aumentó significativamente al primer control $(4,35$ a $4,9 \mathrm{mEq} / \mathrm{L})$ al igual que la creatinina plasmática $(0,9$ a $1,1 \mathrm{mg} / \mathrm{dL})$. Siete pacientes desarrollaron hiperkalemia $(30,4 \%)$. Se suspendió cotrimoxazol en 10 casos $(43,5 \%)$ y en 6 casos se postergó el alta. El uso de fármacos activos contra el sistema renina-angiotensina (FASRA) se asoció a hiperkalemia (OR 10,8 $\mathrm{IC}_{95}$ 1,37-85; p < 0,05). La estadía hospitalaria fue mayor en el grupo con toxicidad a cotrimoxazol (mediana de 56 versus 30 días; $\mathrm{p}<0,05$ ) y los pacientes sin suspensión de terapia tuvieron menos gastos por fármacos (medianas de 563 vs 2.820 USD, $\mathrm{p}<0,01)$. Conclusiones: El uso de cotrimoxazol debe ser monitorizado para detectar hiperkalemia o toxicidad renal y suspender su prescripción. Los pacientes que usan FASRA tienen mayor riesgo de hiperkalemia. La estadía y gastos hospitalarios por fármacos son menores en pacientes que toleran el cotrimoxazol.

\section{Referencias bibliográficas}

1.- Grim S A, Rapp R P, Martin C A, Evans M E. Trimethoprim-sulfamethoxazole as a viable treatment option for infections caused by methicillin-resistant Staphylococcus aureus. Pharmacotherapy 2005; 25: 253-64.

2.- Goldberg E, Paul M, Talker O, Samra Z, Raskin M, Hazzan R, et al. Co-trimoxazole versus vancomycin for the treatment of methicillin-resistant Staphylococcus aureus bacteraemia: a retrospective cohort study. J Antimicrob Chemother 2010; 65: 1779-83.

3.- Spellberg B, Lipsky B A. Systemic antibiotic therapy for chronic osteomyelitis in adults. Clin Infect Dis 2012; 54: 393-407.

4.- Fraser T N, Avellaneda A A, Graviss E A, Musher D M. Acute kidney injury associated with trimethoprim/sulfamethoxazole. J Antimicrob Chemother 2012; 67: 1271-7.

5.- Alappan R, Perazella M A, Buller G K. Hyperkalemia in hospitalized patients treated with trimethoprim-sulfamethoxazole. Ann Intern Med 1996; 124: 316-20.

6.- Antoniou T, Gomes T, Juurlink D N, Loutfy M R, Glazier R H, Mamdani M M. Trimethoprimsulfamethoxazole-induced hyperkalemia in patients receiving inhibitors of the reninangiotensin system: a population-based study. Arch Intern Med 2010; 170: 1045-9.
7.- Gentry C A, Nguyen A T. An evaluation of hyperkalemia and serum creatinine elevation associated with different dosage levels of outpatient trimethoprim-sulfamethoxazole with and without concomitant medications. Ann Pharmacother 2013; 47: 1618-26.

8.- Nguyen S, Pasquet A, Legout L, Beltrand E, Dubreuil L, Migaud H, et al. Efficacy and tolerance of rifampicin-linezolid compared with rifampicin-cotrimoxazole combinations in prolonged oral therapy for bone and joint infections. Clin Microbiol Infect 2009; 15: 1163-9.

9.- Stein A, Bataille JF, Drancourt M, Curvale G, Argenson JN, Groulier P, et al. Ambulatory treatment of multidrug-resistant Staphylococcusinfected orthopedic implants with highdose oral co-trimoxazole (trimethoprimsulfamethoxazole). Antimicrob Agents Chemother 1998; 42: 3086-91.

10.- Greenberg S, Reiser I W, Chou S Y, Porush J G. Trimethoprim-sulfamethoxazole induces reversible hyperkalemia. Ann Intern Med 1993; 119: 291-5.

11.- Greenberg S, Reiser I W, Chou S Y. Hyperkalemia with high-dose trimethoprimsulfamethoxazole therapy. Am J Kidney Dis 1993; 22: 603-6.

12.- Zietse R, Zoutendijk R, Hoorn EJ. Fluid, electrolyte and acid-base disorders associated with antibiotic therapy. Nat Rev Nephrol 2009; 5: 193-202.

13.- Schlanger L E, Kleyman T R, Ling B N. K+ -sparing diuretic actions of trimethoprim: inhibition of $\mathrm{Na}+$ channels in $\mathrm{A} 6$ distal nephron cells. Kidney Int 1994; 45: 1070-6.

14.- Eiam-Ong S, Kurtzman N A, Sabatini S. Studies on the mechanism of trimethoprim-induced hyperkalemia. Kidney Int 1996; 49: 1372-8.

15.- Ho J M, Juurlink D M. Considerations when prescribing trimethoprim-sulfamethoxazole. Can Med Assoc J 2011; 183: 1851-8.

16.- Antoniou T, Gomes T, Mamdani M M, Yao Z, Hellings C, Garg A X, et al. Trimethoprimsulfamethoxazole induced hyperkalaemia in elderly patients receiving spironolactone: nested case-control study. Br Med J 2011; 343: d5228.

17.- Antoniou T, Hollands S, Macdonald E M, Gomes T, Mamdani M M, Juurlink D N, et al. Trimethoprim-sulfamethoxazole and risk of sudden death among patients taking spironolactone. Can Med Assoc J 2015; 187: E138-43.

18.- Fralick M, Macdonald E M, Gomes T, Antoniou T, Hollands S, Mamdani M M, et al. Co-trimoxazole and sudden death in patients receiving inhibitors of renin-angiotensin system: population based study. Br Med J 2014; 349: g6196.

19.- Kutscha-Lissberg F, Hebler U, Muhr G, 
Köller M. Linezolid penetration into bone and joint tissues infected with methicillin-resistant Staphylococci. Antimicrob Agents Chemother 2003; 47: 3964-6.

20.- Rayner C R, Baddour L M, Birmingham M C, Norden C, Meagher A K, Schentag J J. Linezolid in the treatment of osteomyelitis: Results of compassionate use experience. Infection 2004; 32: 8-14.

21.- Senneville E, Legout L, Valette M, Yazdanpanah Y, Beltrand E, Caillaux M, et al. Effectiveness and tolerability of prolonged linezolid treatment for chronic osteomyelitis: A retrospective study. Clin Ther 2006; 28: 1155-63.

22.- Falagas M E, Siempos II, Papagelopoulos P J, Vardakas K Z. Linezolid for the treatment of adults with bone and joint infections. Int $\mathrm{J}$ Antimicrob Agents 2007; 29: 233-9.

23.- Jover-Díaz F, Talents A, Alcalá-Santaella R, Cuadrado J M, Olmedo N, Gazquez C.
Efficacy and tolerability of linezolid therapy in the treatment of orthopedic implant infections caused by gram-positive multiresistant cocci. Rev Clin Esp 2010; 210 145-8.

24.- Lipsky B A, Itani K, Norden C. Linezolid Diabetic Foot Infections Study Group. Treating foot infections in diabetic patients: A randomized, multicenter, open-label trial of linezolid versus ampicillin-sulbactam/ amoxicillin-clavulanate. Clin Infect Dis 2004; 38: 17-24.

25.- Watkins R R, Lemonovich T L, File T M Jr. An evidence-based review of linezolid for the treatment of methicillin-resistant Staphylococcus aureus (MRSA): place in therapy. Core evidence 2012; 7: 131-43.

26.- Senneville E, Legout L, Valette M, Yazdanpanah Y, Giraud F, Beltrand E, et al. Risk factors for anaemia in patients on prolonged linezolid therapy for chronic osteomyelitis: a case-control study. J Antimicrob Agents 2004; 54: 798-802.

27.- Landersdorfer C B, Bulitta J B, Kinzig M, Holzgrabe U, Sörgel F. Penetration of antibacterial into bone. Pharmacokinetics, pharmacodynamic and bioanalytical consideration. Clin Pharmacokinet 2009; 48: 89124.

28.- Doi Y, Chambers H F. Penicillins and $\beta$-lactamase inhibitors. In: Bennett J E, Dolin R, Blaser M J, editors. Mandell, Douglas, and Bennett's Principles and Practice of Infectious Diseases. $8^{\text {th }}$ ed. Philadelphia: Elsevier Saunders; 2015, p. 263-92.

29.- Euba G, Murillo O, Fernández-Sabé N, Mascaró J, Cabo J, Pérez A, et al. Long-term followup trial of oral rifampicin-cotrimoxazole combination versus intravenous cloxacillin in treatment of chronic staphylococcal osteomyelitis. Antimicrob Agents Chemother 2009; 53: 2672-6. 